\title{
Acute Kidney Injury Is Important in the Hospital and Afterward
}

\author{
Benjamin J. Lee, MD ${ }^{1 *}$, Chi-yuan Hsu, MD, MSc ${ }^{1}$
}

${ }^{1}$ Division of Nephrology, Department of Medicine, University of California San Francisco, San Francisco, California.

Acute kidney injury (AKI) is a major contributor to morbidity and mortality in hospitalized patients across the world. ${ }^{1}$ Affecting up to $20 \%$ of all admissions (depending on which definition of AKI is used), ${ }^{2} \mathrm{AKI}$ is the most common reason for new-inpatient nephrology consultation. Recent data suggest that AKI incidence has risen rapidly, by up to $10 \%$ per year. ${ }^{3,4}$

$\mathrm{AKI}$ is associated with a variety of serious short- and longterm complications. Approximately $33 \%$ to $60 \%$ of critically ill patients who develop dialysis-requiring AKI do not survive to hospital discharge, and mortality associated with dialysis-requiring $\mathrm{AKI}$ is greater than that associated with other serious conditions such as myocardial infarction or acute respiratory distress syndrome. ${ }^{5}$ Even relatively mild AKI in the acute inpatient setting appears to be an independent risk factor for mortality. ${ }^{6}$

For several decades, many physicians believed that AKI was a self-limited process followed by complete recovery of renal function to pre-AKI levels among survivors. (Numerous trainees have been taught some variant of the old adage: "If the patients survive, so will their kidneys.") But studies linking AKI with the development of new-onset chronic kidney disease (CKD) or the accelerated progression of pre-existing CKD have changed this view. ${ }^{7}$ One important reason the long-term impact of AKI hasn't been appreciated is that, traditionally, clinical studies of AKI examined inhospital outcomes such as short-term mortality and resource usage and did not consider what transpired months to years after discharge. More recently, epidemiologic studies linking inpatient events with outpatient outcomes have filled this knowledge gap. ${ }^{8}$ Contemporary animal models of AKI have shed light on potential mechanisms of maladaptive repair after AKI, characterized by fibrosis, vascular rarefaction, tubular loss, glomerulosclerosis, and chronic interstitial inflammation, all of which result in renal function decline. So over the last decade there has been a paradigm shift in how we think about AKI and CKD. Rather than distinct entities, $\mathrm{AKI}$ and $\mathrm{CKD}$ are now viewed as interconnected syndromes since $\mathrm{AKI}$ is a risk factor for CKD progression and CKD is a risk factor for new episodes of AKI. ${ }^{9}$

Two studies published in this issue of the Journal of Hospital Medicine augment our understanding of AKI and its clin-

\footnotetext{
*Address for correspondence and reprint requests: Benjamin J. Lee, MD, Division of Nephrology, University of California San Francisco, 533 Parnassus Avenue, U404, San Francisco, CA 94143; Telephone: 415-476-1812; Fax: 415476-3381; E-mail: bjlee@ucsf.edu
}

Received: September 26, 2016; Accepted: October 9, 2016

2017 Society of Hospital Medicine DOI 10.12788/jhm.2691 ical impact in hospitalized patients. Analyzing data from the National Inpatient Sample, Silver et al. ${ }^{10}$ found that hospitalizations that include AKI are substantially costlier and associated with longer lengths of stay than hospitalizations without AKI. The authors also highlight that the additional economic costs of AKI exceeded those of many other higher-profile yet less-common acute medical conditions, such as myocardial infarction and gastrointestinal bleeding. These results re-emphasize the important economic burden of AKI at a national level and expand on prior literature by confirming findings previously limited to single-center and regional studies. Better defining the impact AKI has on our healthcare system could help ensure that adequate resources are invested to combat AKI.

The second study, by Rutter et al., ${ }^{11}$ found that among hospitalized patients with normal baseline renal function, use of vancomycin in combination with piperacillin-tazobactam is associated with a higher incidence of AKI after antibiotic exposure than use of either agent as monotherapy. This association persisted even after adjusting for potential confounders such as underlying comorbidities, exposure to nephrotoxic agents, documented hypotension, and baseline renal impairment. This study adds to a growing body of literature that suggests synergistic nephrotoxicity between vancomycin and piperacillin-tazobactam. It underscores that any medical intervention-even treatments typically envisioned as non-hazardous and frequently life-saving - involve inherent risks and should prompt the medical community to promote proper antimicrobial stewardship. Whether such exposures to vancomycin or beta-lactam derivatives cause AKI via direct tubular damage, interstitial nephritis, or some other novel mechanism remains to be elucidated. Better delineation of the contemporary causes of AKI, including increased antibiotic exposure, is the first step toward identifying ways to reduce AKI incidence.

Both of these papers serve to highlight the clinical importance of AKI among hospitalized patients. Their findings re-emphasize the need for vigilance in detecting AKI and intervening early to achieve the best clinical outcomes.

Given recent understanding that survivors of AKI are at greater risk for more rapid loss of renal function long after hospital discharge, one goal the US Department of Health and Human Services put forth for Healthy People 2020 is to "increase the proportion of hospital patients who incurred AKI who have follow-up renal evaluation in 6 months post-discharge" (10\% improvement targeted). ${ }^{12}$ Transitions of care after hospitalizations complicated by AKI require special attention to ensure that patients' needs are optimally 
monitored and managed during the critical post-discharge period. One recent study analyzing discharge documentation for hospitalizations including AKI found that fewer than half of the discharge summaries and patient instructions commented on the presence, cause, or course of AKI, indicating clear room for improvement. ${ }^{13}$ And currently, it appears that only a minority of patients with AKI-even AKI severe enough to require dialysis_-are seen by a nephrologist within 90 days of discharge. ${ }^{14}$

Hospitalists play a crucial role in coordinating care as vulnerable patients transition from the inpatient to outpatient setting. We suggest that AKI should be properly documented in the discharge summary. In addition, patients should be informed that they experienced AKI so they can discuss with future caregivers potential strategies to avoid additional renal insults. Discharge referrals to nephrology should be arranged for high-risk patients, including those whose renal

\section{References}

1. Lameire NH, Bagga A, Cruz D, et al. Acute kidney injury: an increasing global concern. Lancet. 2013;382(9887):170-179.

2. Zeng X, McMahon GM, Brunelli SM, Bates DW, Waikar SS. Incidence, out comes, and comparisons across definitions of AKI in hospitalized individuals. Clin J Am Soc Nephrol. 2014;9(1):12-20.

3. Hsu RK, McCulloch CE, Dudley RA, Lo LJ, Hsu CY. Temporal changes in incidence of dialysis-requiring AKI. J Am Soc Nephrol. 2013;24(1):37-42.

4. Siew ED, Davenport A. The growth of acute kidney injury: a rising tide or just closer attention to detail? Kidney Int. 2015;87(1):46-61.

5. Cerdá J, Liu KD, Cruz DN, et al. Promoting kidney function recovery in patients with AKI requiring RRT. Clin J Am Soc Nephrol. 2015;10(10):1859-1867.

6. Chertow GM, Burdick E, Honour M, Bonventre JV, Bates DW. Acute kidney injury, mortality, length of stay, and costs in hospitalized patients. J Am Soc Nephrol. 2005;16(11):3365-3370.

7. Hsu CY. Yes, AKI truly leads to CKD. J Am Soc Nephrol. 2012;23(6):967-969.

8. Coca SG, Singanamala S, Parikh CR. Chronic kidney disease after acute kidney function remains decreased at discharge or those who had recurrent AKI episodes during prior hospitalizations. For patients with pre-hospitalization baseline CKD, nephrology should be consulted before indefinitely discontinuing medications like angiotensin-converting enzyme inhibitors or angiotensin receptor blockers. These medications are indispensable in retarding the progression of proteinuric CKD, even though they may predispose patients to AKI under certain circumstances (eg, in states of decreased renal perfusion). Adopting these simple steps may substantially improve the long-term outcomes of patients who experience AKI during hospitalization.

\section{Acknowledgments}

The authors are supported by NIH-NIDDK Grants T32DK007219 (BJL) and K24DK92291 (CYH).

Disclosure: Nothing to report.

injury: a systematic review and meta-analysis. Kidney Int. 2012;81(5):442-448

9. Chawla LS, Eggers PW, Star RA, Kimmel PL. Acute kidney injury and chronic kidney disease as interconnected syndromes. New Engl J Med. 2014;371(1):58-66.

10. Silver SA, Long J, Zheng Y, Chertow GM. Cost of acute kidney injury in hospitalized patients. J Hosp Med. 2017;12(2):70-76.

11. Rutter WC, Burgess DR, Talbert JC, Burgess DS. Acute kidney injury in patients treated with vancomycin and piperacillin-tazobactam: a retrospective cohort analysis. J Hosp Med. 2017;12(2):77-82.

12. US Department of Health and Human Services, Office of Disease Prevention and Health Promotion. Healthy People 2020. Available at: https://www.healthypeople.gov/node/4093/data_details. Accessed September 2, 2016

13. Greer RC, Liu Y, Crews DC, Jaar BG, Rabb H, Boulware LE. Hospital discharge communications during care transitions for patients with acute kidney injury: a cross-sectional study. BMC Health Serv Res. 2016;16:449.

14. Siew ED, Peterson JF, Eden SK, et al. Outpatient nephrology referral rates after acute kidney injury. J Am Soc Nephrol. 2012;23(2):305-312. 